\title{
DE LAS "IDEAS" A LAS “CIFRAS" EN LA FILOSOFÍA DE JASPERS *
}

La Philosophie de Karl Jaspers se organiza en torno a tres polos que revelan inequívocamente su filiación kantiana, puesto que reiteran los interrogantes que, como decia Kant, resumen "todo el interés de mi razón (tanto de la especulativa como de la práctica)": " $x$ ) ¿Qué puedo saber? 2) ¿Qué debo hacer? 3) ¿Qué me está permitido esperar?"." Las tres partes de la mencionada obra de Jaspers, en efecto, apuntan a esas preguntas, y se basan en "impulsos existenciales" expresables como "máximas": 1) La Orientación en el mundo establece lo que puedo saber y lleva a los límites de lo cognoscible. "El mundo debe ser conocido para ver lo que es el ser". 2) La Aclaración de la existencia se mueve en la dimensión ya no cognoscible donde sólo tiene sentido la apelación a la libertad de quien actúa: "Depende de mi". Por último, 3) la Metafísica examina los diversos modos en que puedo esperar "hacerme presente" la Trascendencia: "Yo puedo buscar a Dios." 2 Las tres partes' no tienen sentido aisladamente; cada una prepara y reclama las otras; un único movimiento las atraviesa a todas, pues "sin existencia, la orientación en el mundo no tendría sentido y la Trascendencia se convertiría en superstición. Sin orientación en el mundo, la existencia llegaria a ser puntualidad vacia y la Trascendencia permanecería sin la materia de un lenguaje. Sin Trascendencia, la existencia perdería su propio ser-sí-mismo, y la orientación en el mundo su posible profundidad. El hombre es posible existencia que, como conciencia en general, se orienta en el mundo y, a través del mundo, está referido a la Trascendencia".s

- Este trabajo resume la parte central de una obra más ambiciosa, aún inédita, donde trazo un cuadro general de la filosofia de Jaspers, tratando siempre de ordenarla en torno a su peculiar interpretación de la filosofia de Kant. Estudio alli, en primer término, las confrontaciones con Rickert y Husserl, que llevaron a Jaspers a su idea de la filosofia; luego establezco las distinciones entre Jaspers, Heidegger y Marcel. Finalmente, paso a exponer los puntos principales de su filosofía.

1 Kritik der reinen Vernunft, A 804 s.; B 832 s.

2 Philosophie, Berlín-Göttingen-Heidelberg, $3^{a}$ ed., 1956, t. 1, p, 52.

3. Ibid. Es importante sefialar que esta irrupción de la Trascendencia en la filosofia de Jaspers no acontece en virtud de una prueba - pues ello quedaría en el plano del saber, es decir, para él, del objeto empírico -, ni tampoco debido a algín tipo de derivación o desarrollo, cualquiera sea, que haga surgir la certeza de la Trascendencia a partir de la existencia o del conocimiento del mundo. Antes bien, como observan Dufrenne y Ricoeur, "desde las primeras páginas de la Philosophie es nombrada la Trascendencia y está ya presente como aquello mismo que busca la filosofia". Con un solo movimiento "se propone un edificio de tres plantas; no se encontrará en la obra de Jaspers con qué justificar el último salto" (de la libertad a la Trascendencia). La filosofia no puede sino despertar en nosotros la necesidad de reconocerse frente a lo absoluto, Cfr. Dufrenne y Ricoeur, Karl Jaspers et la philosophie de l'existence, Paris, 1947, p. 298, n. 7 . 
Sólo a partir de su filiación kantiana, y a despecho de las interpretaciones corrientes que lo exponen únicamente como "existencialista", es posible comprender el desarrollo de la filosofía de Jaspers - desarrollo que, como veremos, ciertamente la separa cada vez más de su raíz kantiana para aproximarla inevitablemente al pensamiento de Kierkegaard, Entre quienes destacan esta línea interpretativa, debemos mencionar a Stegmüller. Atinadamente observa éste que uno de los supuestos del pensamiento de Jaspers es "una amplia aceptación de la posición gnoseológica de Kant", y no sólo de sus puntos de partida, sino "también de los últimos resultados de la teoría del conocimiento de Kant, especialmente del idealismo trascendental y de la teoría de la incognoscibilidad del ente en si". La tẹnsión entre Kant y Kierkegaard de que hablábamos, la polaridad Razón y Existencia, surge en la medida en que la filosofía crítica sostiene puntos de vista "racionalmente discutibles, que tienen que ser verdaderos o falsos en el sentido de la verdad cientifica", mientras que Jaspers "trata de relativizar todo lo científicamente cognoscible desde una 'más elevada atalaya', y traslada la verdad más profunda que se puede alcanzar a la vivencia existencial del individuo"."

Para rastrear la singular interpretación de Kant que está a la base del filosofar de Jaspers consideramos oportuno, pues, seguir la línea que comienza por la orientación en el mundo, choca con los límites del saber, recurre a las "Ideas" y las transforma en el concepto de das Umgreifende - palabra que traduciremos, según el contexto, como "lo englobante" o "lo abarcador"- - Veremos finalmente cómo este movimiento concluye con la negación de la filosofía en cuanto trasmisión objetiva de un contenido pensado, y en su recuperación como "cifra" de una experiencia intransferible, sólo "descifrable" por el individuo histórico.

\section{La orientación en el mundo}

El hombre que despierta a la vida consciente, en su situación, trata de orientarse en el mundo. La situación actual no puede prescindir de las ciencias ya que siempre han interpretado la realidad; sin ellas no tendría lugar una posterior orientación filosófica en el mundo, afirma Jaspers.

En primer lugar es menester recordar que todo conocimiento objetivo supone el "principio de la conciencia", es decir, el factum de que al conocer estamos en la escisión sujeto-objeto. ${ }^{5}$ En la vida cognoscitiva no podemos

4 Stegmüller, Hauptströmungen der Gegenwartsphilosophie, Stuttgart, $3^{\text {a }}$ ed. muy ampliada, 1965, pp. 233 ss. Stegmüller señala lo paradójico de esta situación, que vemós en el desarrollo de nuestra exposición: para su metodologia, Jaspers supone no sólo los planteamientos iniciales sino también los resultados finales de una "filosofía cientifica" que, de acuerdo con su propia idea de la filosoffa, es absolutamente imposible, cfr. ibid., p. 235 .

5 Jaspers habla casi siempre de "escisión sujeto-objeto", y no de "relación", ya que esto último supondrfa la existencia de dos cosas separadas, mientras que la primera deno. 
salir de esa situación; sea lo que fuere que conozcamos de ella, siempre te nemos que pensarla de modo objetivo, presuponiendo así la misma relación que se trata de pensar. Es, como diría Marcel; un problema "qui empiète sur ces propres données, qui les envahit et se dépasse par là même comme simple probleme". ${ }^{6}$ La aprehensión directa de tal escisión exigiría ir más allá de toda objetividad; y esto es precisamente lo que, según Jaspers, ha hecho Kant: señalar las condiciones de posibilidad de lo objetivo significa,' en efecto, trascender hacia algo no objetivo. Kant "cambió la dirección del trascender; (...) llevó a cabo lo que denominó método trascendental, y lo diferenció de un trascender a un ser-cosa situado en el más allá, pero manteniendo sin embargo el trascender como tal, por cuya virtud toda existencia empírica se convirtió en fenómeno (Erscheinung)".?

Ahora bien, la misma enunciación de ese acto de trascender y de aquello a que "llega", recae necesariamente en formulaciones objetivantes. Por ello, acentúa Jaspers, Kant se esforzó enormemente (especialmente en el capítulo sobre la "deducción trascendental", el que consideraba el más profundo de su primera Critica) para que el lector fuera capaz de realizar por sí mismo el auténtico trascender; pues si se entiende deficientemente este nuevo y revolucionario modo de pensar, continúa, uno podria creer que "posee" el a priori, la unidad trascendental de la apercepción, etc. como si se tratara de conceptos fijos y definibles de algo; pero el verdadero acto de trascender se logra en el límite, en el paso mismo de lo objetivo a lo inobjetivable. Entonces se comprende que aquellos conceptos son funciones, y no intelecciones o conocimientos. La "objetivación" de los enunciados kantianos, su interpretación psicológico-antropológica y la metodológico-gnoseológica, por ejemplo, anulan el mismo movimiento trascendente; la primera, haciendo surgir el mundo de la estructura de la organización cerebral y anímica del hombre; la segunda, en cuanto hace de las condiciones metodológicas del conocimiento de las cosas las condiciones de la existencia real de los objetos experimentados. Ambas razonan en círculo, afirma Jaspers: el cerebro crea el mundo, del cual es a su vez parte y producto; los métodos crean los objetos, en cuyo conocimiento únicamente se desarrollan, de suerte que están condicionados por éstos.

La revolución copernicana efectuada por Kant, según Jaspers, se resume en la siguiente afirmación: "Todos los objetos son solamente fenómenos; ningún ser conocido es el ser en sí y en su totalidad. El carácter fenoménico de la existencia empírica es una intelección básica del pensar filosófico. Si

minación -usada en la escuela neokantiana de Baden- señala el desgarramiento de una unidad originaria, apunta a la dimensión de lo no escindido. Cfr. Hans Saner, Karl Jaspers in Selbstzeugnissen und Bilddokumenten, Hamburgo, 197o, p. 85 .

B Gabriel Marcel, Position et approches concrètes du Mystère ontologique, LouvainParis, 1949, p. 57.

7 Philosophie, I, pp. 40 s. 
bien dicha intelección no es comprensible de modo objetivo, sino únicamente por el acto de trascender, no puede sustraerse a ella ningún entendimiento que en general sea capaz de trascender. [...] Ella no agrega un nuevo saber particular al saber hasta entonces obtenido, sino que provoca una sacudida de la conciencia del ser en su totalidad." 8

Ahora se comprende por qué, antes de trascender en el modo de la libertad y de la metafísica, Jaspers despliega la orientación que trasciende sobre la totalidad del mundo, o mejor dicho, sobre el mundo como totalidad. El ser conocido por las ciencias no agota la riqueza del ser; en caso contrario, el saber científico expresaría la última verdad; el mundo existirfa como ser en si. "Tan sólo la orientación filosófica en el mundo, al romper la oclusión de éste y al hacerme retroceder hacia mí mismo, posibilita que yo llegue a estar abierto para la Trascendencia." "

El mundo es Erscheinung; pero aquello de que él sería manifestación no es por principio ninguna objetividad; ni siquiera es en si un ser determinado. La fenomenicidad del mundo expresa la "suspensión" en que el trascender, en la conciencia del límite, coloca al mundo y a mí como ser en el mundo (pero sin sacarme de él para llevarme a un más allá). Por ello, dice Jaspers, la misma expresión "el mundo es fenómeno", no tiene sentidoen el mundo: "este sentido proviene sólo del límite"."

Por otra parte, las ciencias que investigan los entes que se dan en ek mundo tampoco poseerían unidad metódica - puesto que no es dada la unidad del mundo - si ésta no proviniera también de otro origen. Ninguna ciencia tiene por objeto el mundo como totalidad y unidad, pues ello contradice el principio del conocimiento; pero toda ciencia tiende a la unidad del mundo entendida no como dada (gegeben), sino como tarea (aufgegeben, Aufgabe) del constante progreso de la investigación. Pero este "resultado" no hace sino retomar lo que Kant entendia por Idea. Y tal es, en efecto, la conclusión de esta primera parte de la Philosophie: "Kant comprendió que el mundo no llega a ser objeto para nosotros, sino que sólo es una Idea; esto es, que todo lo que podemos conocer es en el mundo, pero jamás el mundo; y que, cuando hipotéticamente pretendemos conocer el mundo como totalidad en sí entitativa, nos enredamos en contradicciones insolubles - las antinomias_." 11

\section{Hacia los limites de la razón}

Más tarde, al transcribir en el primer tomo de su Lógica filosófica, casi textualmente, el párrafo anterior, Jaspers reemplaza la palabra "Idea" con

8 Der philosophiesche Glaube, München, $2^{\text {a }}$ ed., 1951, p. 32.

- Philosophie, I., pp. 30 s.

10 Philosophie, I., p. 43 .

11 Existenzphilosophie, Berlin, 2* ed., 1956, pp. 15 s. 
un nuevo concepto que apunta ya a la teoria de las cifras. Dice alli: "Kant comprendió (que) el mundo es tan sólo Idea [...]: El mundo es lo englobante en el cual y desde el cual nos hace frente todo ser mundanal. [...] El ser-Idea del mundo es expresión de su ser-englobante (Umgreifend-sein)." 18 La filosofía, por ende, para pensar el mundo sin hipostasiarlo, no puede sino realizar la "inversión" que Kant formuló con claridad por vez primera, y cuyo enunciado reza, paradójicamente: "Pensar (en) los objetos, pero de modo no objetivante; en el acto de pensar, saltar sobre la propia sombra; con el entendimiento, pensar con métodos que van más allá del entendimiento", ${ }^{18}$ pues la filosofía es cosa de la razón.

La nada ortodoxa manera en que Jaspers recurre a Kant nos fuerza a detenernos en algunos pormenores. Recuerda Jaspers que el neokantismo habia popularizado dos consignas: "Hay que volver a Kant" (Liebmann) y "Comprender a Kant significa ir más allá de Kant" (Windelband). Pero en filosofía - sostiene Jaspers - no se trata de "volver", como si hubiera que poner nuevamente en circulación verdades definitivas y objetivas, ni tampoco se trata de "superar" un momento histórico determinado: la vuelta a Kant no significaria retroceder, sino internarse en el origen; y "más alla" no puede significar un "saber mejor", sino solamente el movimiento del trascender que es el corazón mismo del pensamiento kantiano.14 Pero para conciliar asi las dos máximas, en primer lugar, habria que depurar a Kant de la interpretación de los mismos neokantianos, pues éstos generalmente vieron su filosofía como una mera "teoria del conocimiento", y ello es "un tardío empobrecimiento y trivialización del filosofar de Kant". ${ }^{15}$

Según Jaspers, una interpretación más profunda de Kant debe centrarse en primer lugar sobre el sentido de las antinomias, entendidas como signos dè una realidad no objetivable que se hace presente, de modo indirecto, en los enunciados objetivos. En segundo lugar, hay que concebir la razón con la amplitud con que la concibió el mismo Kant, y no con la estrechez "racionalista". Con respecto a lo primero, aclara: "Por cuanto lo inobjetivo que debe ser aclarado en el origen de todo ser objetivo (en la escisión sujetoobjeto) sólo puede ser pensado, empero, objetivamente, lo así pensado - para que no se consolide de modo falso como si fuera un objeto- tiene que fracasar formalmente en tautologías, circulos y contradicciones." 18 Este punto se complementa en el segundo, que muestra el interés de Jaspers por rescatar del racionalismo estrecho los límites irracionales en que, según él, se movep. $4^{8}$.

12 Von der Wahrheit. Philosophische Logik. Erster Band, München, Neuausgabe, 1958,

18 "Antwort" de Jaspers, en P. A. Schilpp (ed.), Karl Jaspers, Stuttgart, 1957, p. 79a. 14 Die grossen Philosophen (1957), reeditado como Plato, Augustin, Kant. Drei Grinder des Philosophierens, München, 1967, p. 896.

15 Von der Wahrheit, p. 22.

10 Die grossen Philosophen, p. 226. 
ría el pensar de Kant -lo que, además, permitiría la inestable conciliación de las antinomias kantianas con las paradojas de Kicrkegaard--.

No hay que olvidar, "en este respecto, la insistencia con que Jaspers - ya desde su juvenil informe para el seminario de Emil Lask, incluido como apéndice a la Psychologie der Weltanschauungen - destaca el limite irracional que pertenece esencialmente a la actitud racional, cosa que pondría en evidencia la teoría de las Ideas de Kant. Así considerada, "la filosofía kantiana es el racionalismo que se sostiene y supera a sí mismo". ${ }^{17}$ Por de pronto - escribirá en su conferencia sobre "El mal radical en Kant" (1935)- es preciso darse cuenta de que la razón no se agota en el entendimiento que piensa objetivamente en virtud de las categorias, sino que es además la facultad que por las Ideas da unidad sistemática a lo particular dentro de la totalidad, y es también el fundamento del obrar moral y la base de la intuición de lo bello. La filosofía de Kant, en tal sentido, es para Jaspers una única y grandiosa aclaración de la razón en todos sus dominios -teórico, práctico y estético. Pero es una aclaración que en cada ámbito choca contra lo otro a que está remitida la razón misma, con los límites. Pues de lo contrario la razón quedaría inconcebible en su mismo origen. Por ello, insiste, el filosofar de Kant está siempre acompañado de "enigmas" que él, en cada caso, fue el primero en expresar con claridad. (El "mal radical" es precisamente uno de los más fundamentales enigmas de su filosofía.) La paradoja de ese filosofar, por tanto, reside en el hecho de que, iluminando la razón en todas sus formas, "con apasionada racionalidad", lleva à la misma razón a cerciorarse de sus límites. Al saberse abarcada, la razón no está por ello lïmitada en su ejecución, pues el mismo englobante es accesible tan sólo. gracias a la razón que lo ilumina. Así, pues, destacar lo englobante irracional que circunda a la razón no implica "perder la razón", porque en tal caso con ella se perdería la misma experiencia del límite, y lo que está más allá del límite y la abarca. ${ }^{18}$

Este enfoque, dice Jaspers, quiere ponernos en claro sobre el hecho de que vivimos, "en la prisión de la objetividad" - pero la misma filosofía de Kant, al mostrar la prisión como tal, ejerce un acto liberador. ${ }^{19}$ En los límites de lo objetivamente cognoscible, la existencia se ve compelida a trascender el saber para dar testimonio del insondable origen del conocimiento, del abrar y de la contemplación. Esta última conversión (que en Jaspers

17 Von der Wahrheit, p. 22.

18 Cfr. "Das radikal Böse bei Kant", en Rechenschaft und Ausblick, München, 1951, pp. $105-109$.

19 Cfr. Die grossen Philosophen, pp, $198 \mathrm{~s}$. Es evidente aquil la influencia de ciertas ideas de Simmel, de su Lebensanschautung. No en vano, pues, Rickert abarcaba a Jaspers al criticar a la filosofia de la vida como "moda" de la época. Cfr. Heinrich Rickert, Die Philosophie des Lebens. Darstellung und Kritik der philosophischen Modeströmungen unserer Zeit, Tübingen, $2^{\text {a }}$ ed., 1922. 
se llamará "fe filosófica") toma al pie de la letra la confesión de Kant, en el Prólogo a la segunda edición de la Crítica de la razón pura: "Tuve, por tanto, que suprimir el saber, para obtener lugar para la fe." 20

Ahora bien, si retrocedemos un poco en nuestra exposición, veremos hasta qué medida Jaspers, traicionando su punto de partida kantiano, se aproxima cada vez más al polo existencial. Si atendemos por ahora sólo al aspecto teórico del problema - tal como se lo enfoca en la Orientación en el mundo- puede precisarse que, mientras Kant demuestra la inaplicabilidad de las categorías a las cosas en sí basándose en argumentaciones racionales (esto es, "científicas", aunque le pese a Jaspers), éste por el contrario, sobrepasa lo racional ya con su propio "método". En efecto, y a diferencia de Kant, en Jaspers ni siquiera puede saberse en qué reside la incognoscibilidad del mundo real - como acertadamente lo enuncia Stegmüller_, ya que ese mismo saber acerca del fundamento de la incognoscibilidad sería a su vez objetivo y obligatorio, sería "ontológico" (y Jaspers niega la posibilidad de la ontologia). En lugar de una fundamentación lógica de los límites del saber científico, da Jaspers numerosos ejemplos de límites fácticos. Pero la meta de ese procedimiento no sería la solución de cuestiones gnoseologicas fundamentales (como hace Kant), sino "el descubrimiento del desgarramiento, la disarmonía y la cuestionabilidad inherentes al mundo en el cual el pensador solitario se encuentra puesto en inaclarables situaciones, sin hallar sosiego en conocimientos de validez universal". ${ }^{21}$

\section{La interpretación de las Ideas}

El Referat para el seminario de Lask no sólol documenta el temprano interés de Jaspers por la filosofía de Kant, sino también la peculiaridad de su enfoque, centrado en la teoría de las Ideas. Esto, señala Gerhard Knauss, era novedoso frente a los estudios corrientes en esa época, limitados más bien a los problemas de la deducción trascendental y de las categorias. ${ }^{22}$ Tanto en dicho informe, titulado precisámente "Kants Ideenlehre" como en la obra en que fue publicado como apéndice algunos años más tarde, la $P$ hychologie der Weltanschauungen (1919), alude a las Ideas como fuente del movimiento de la razón, a la que aquellas penetran y en cierto modo superan. Es interesante reproducir un oscuro pasaje del final de esa obra, donde se perciben ecos hegelianos y se anticipa la noción de das Umgreifende que Jaspers no explicitaría sino muchos años después. Dice alli: "La auténtica vida de la Idea es movimiento dentro de la escisión sujeto-objeto, es movimiento en lo finito. La vida de la Idea no es dada y cumplida de modo

20 Kritik der reinen Vernunft, $\mathrm{B}$ XXX.

21 Stegmüller, op. cit, pp. $196 \mathrm{ss}$.

22 Cfr. G. Knauss, "Der Begriff des Umgreifenden in Jaspers' Philosophie", en el volumen de P. A. Schilpp ya citado, p. 132. 
inmediato, simplemente como una vivencia, sino mediada por el obrar en el mundo, la experiencia, la reflexión, la meditación sobre sí mismó, etc. Por muy irracional que sea la Idea, ella no se presenta como lo irracional que hace a un lado lo racional, sino que cobra vida en virtud del movimiento infinito en lo racional, penetrándolo y sobreabarcándolo (übergreifend)". ${ }^{23}$

La teoría de las Ideas constituye, pará Jaspers, el núcleo indiscutible que garantiza la auténtica comprensión de la filosofía de Kant y, sobre todo, permite captar la unidad de todas "las facultades del ánimo". A partir de esta interpretación, Jaspers irá elaborando su propia concepción de la filosofía, que culminará con la noción de das Umgreifende. Según Knauss, "el pensamiento fundámental de Kant acerca de la escisión sujeto-objeto, y su 'conclusión' - a partir del reino de la naturaleza y de la libertad- de su 'unidad en el fundamento', más allá de tọa posibilidad de conocimiento (al principio de la Critica del juicio), lo mismo que sus palabras sobre la 'raíz común de la sensibilidad y el entendimiento', invocan de modo casi natural (en Jaspers) la concepción de das Umgreifende". ${ }^{24}$

Jaspers expone de modo casi escolar el papel de las Ideas dentro de la totalidad de la filosofía de Kant. El conocimiento es un proceso estructurado que se inicia con la confusión de las sensaciones, se eleva a las formas puras de la intuición y de allí a las categorias. Estas proporcionan la forma objetivamente determinada de lo cognoscible. Por último, asciende a las Ideas de la unidad sistemática del conocimiento. Este proceso -agrega- es inacabable, no puede concluir en un sistema total; permanece "en suspenso". Ahora bien, en este esquema, las Ideas cumplen la función de testimoniar la irrupción de lo inteligible dentro de la universal objetividad del entendimiento; son, en definitiva, "la presencia de lo suprasensible en la totalidad del conocer". ${ }^{25}$

Aunque Jaspers trata de ser fiel a Kant, distinguiendo inclusive más de una vez la "Idea" kantiana de la "Idea" en su acepción hegeliana, no por ello deja en algunas oportunidades de "hegelianizar" las Ideas, sobre todo al acentuar la objetividad de las mismas -entendiendo, según parece, la validez objetiva de la Idea como si ésta determinara a los objetos-. En tal sentido, no es superfluo señalar que la noción de Idea en Jaspers, como bien lo vieron Dufrenne y Ricoeur -aunque quizá la cuestión no sea en su cómplejidad tan simple como su enunciado- se encuentra siempre en un plano intermedio entre su acepción como principio regulador del conocimiento y como forma más acabada de la realidad objetiva: ${ }^{26}$

23 Psychologie der Weltanschautungen (1919), $4^{\text {a }}$ ed., Berlin-Göttingen-Heidelberg, 1959* p. 460. Traducimos "übergreifend" por "sobreabarcándo", aun cuando la expresión " no es del todo correcta, y mucho menos elegante, para señalar su parentesco con "umgreifen".

24 G. Knauss, op. cit., p. 133 .

25 Die grossen Philosophen, p. 253.

26 Dufrenne y Ricoeur, op. cit., pp. 41-46. 
Dejando a un lado esta cuestión, que nos apartaría de nuestra meta en el presente trabajo, digamos que Jaspers admite tres significaciones -coincidentes, en última instancia - de la nóción de Idea en Kant. La Idea es principio válido para buscar la conexión sistemática del conocimiento (significación metodológica o función heurística); actúa como "fuerza" en la subjetividad del que conoce, ès un germen que impulsa a su propio desarrollo (significación psicológica, ferozmente criticada por Rickert en su comentario acerca de la Psicologia de las concepciones del mundo, en Logos, 9, 1920/21); y finalmente Ia significación objetiva: en la Idea está presente algo que nos sale al encuentro a partir del origen de las cosas. ${ }^{27}$ Esta triple función - confiesa Jaspers en su "Respuesta" a los trabajos a él consagrados, reunidos en el volumen editado por Schilpp- se le hizo patente ya en sus lecturas juveniles de Kant; creyó comprender entonces el procedimiento que éste empleaba: "Kant piensa la Idea, en primer lugar, en su significación objetiva; en segundo lugar, como fuerza motriz subjetiva; en tercer lugar, como origen metodológico de la investigación sistemática. Dentro de esa triple significación, la Idea cambia de sentido de acuerdo con el contexto de las elucidaciones, pero lo hace de modo tal que surge una totalidad dentro de la que la Idea sufre ese necesario cambio de significación. i’ 28

\section{4. 'Hacia la éxpresión indirecta de lo inefable}

Habíamos adelantado ya que Jaspers pasa de una presunta "fidelidad" a la concepción kantiana de las Ideas a una teoría de lo englobante. Aquí sigue en pie la cuestión de cómo sea posible hablar de das Umgreifende sin convertirlo en un objeto -cuestión que nos llevó en otra ocasión a exponer la filosofía de Jaspers confrontándola con Bergson y Wittgenstein. ${ }^{29}$

Es posible hablar de lo englobante, responde Jaspers, pero sólo indirectamente. En efecto, nuestro pensar, aun al referirse a lo que de ningún modo puede ser objeto, ha de enunciarlo siguiendo "el hilo conductor de lo objetivo". Con otras palabras: utilizando expresiones que aparentemente remiten a una realidad objetivamente conocida (o cognoscible), el filósofo, en verdad, apunta a hacer presente de modo alusivo una realidad que por definifición trasciende todo saber objetivo. Y aquí está sin duda la clave de bóveda que sostiene todo el edificio de la metafísica de Jaspers - la cual, es

27 Cfr. Die grossen Philosophen, p. 250.

28 "Antwort", en P. A. Schilpp (ed.), op. cit., pp. $187 \mathrm{~s}$. Refiriéndose a la interpretación del arte en Jaspers, dice Johannes Pfeiffer respecto de las Ideas: "Las Ideas son un abarcador al cual uno no se puede dirigir de modo directo, pero en el cual tiene que vivir de modo activo, o cognoscitivo, o configurativo (en el arte)." "Zur Deutung der Kunst bei $\mathbf{K}$. Jaspers", en Schilpp', op. cit., p. 676 .

29 Cfr. Mario A. Presas; "El estilo del filosofar de Jaspers", en Revista ECO, Bogotá/ Colombia, núm. 159, enero, 1974, pp. 225-237. 
menester insistir en ello, se basa expresamente en una peculiar interpretación de la filosofía de Kant-. El propio Jaspers cuenta que ese modo de "enunciación indirecta" llegó a ser claro para él "por vez primera, en el método de Kant, tal como éste de hecho lo emplea (aunque sin enunciarlo como tal). En su famosa deducción trascendental de la posibilidad de toda objetividad y de todo conocer, Kant procede de tal suerte que, guiándose por objetivaciones psicológicas, lógicas, metodológicas, metafísicas, piensa aquello que no es ninguna de estas objetivaciones, pero si condición de posibilidad de todas ellas; aquello que en si mismo no es sujeto ni objeto. En esto estriba el hecho de que los textos de Kant, alli donde rozan estas cuestiones que según sus palabras constituyen la parte esencial de su filosofía, sean extremadamente difíciles de comprender. [...] Las interpretaciones tienden a tomar uno de los hilos conductores por la cosa misma y, luego, a excluir los otros como modos equivocados de pensar, que Kant no habría superado. Así hubo una interpretación psicológica (Fries), una lógica (Lask), una metodológica (Cassirer), una metafísica (Paulsen)".

"A mí me pareció que el pensamiento trascendental de Kant sólo sería comprendido si se rechazaban esas interpretaciones que conducian a unívocos carriles objetivos, y si uno se precipitaba con Kant en el lenguaje simbólico de múltiples hilos conductores, cada uno de los cuales contradice al otro cuando"se pretende tomarlos como enunciados directos." Este enfoque - cuya exactitud puede ciertamente discutirse— le posibilitó a él mismo, concluye Jaspers, "pensar metódicamente lo englobante". 30

Este método lo lleva a interpretar el lenguaje objetivo en que se trasmiten incluso los pensamientos filosóficos como "cifra". Se ha afirmado que en este punto Jaspers sería más ambicioso que Kant, pues éste llama "problemático y limitativo", al concepto de noumeno (para el cual no disponemos de una adecuada intuición intelectual); sólo la esfera práctica nos brindarian algunas pautas, pero éstas corren por cuenta de la "fe". Jaspers, en cambio, intenta trazar una vía intermedia entre el saber y la fe; las cifras intentarían sustituir a la intuición intelectual ausente; por medio de ellas, y salvando el abismo abierto por Kant, Jaspers querría pensar positivamente el noúmeno. ${ }^{31}$ Sin embargo, al negar a las cifras el carácter de saber, Jaspers permanece fiel a la lección de Kant; "no sacrifica a un nuevo platonismo; no hay mundo de las Ideas (en el sentido de Platón); nada de Dios es conocido fuera de la apariencia múltiple que es su incógnito. La doctrina de las cifras, pues, rebasa pero al mismo tiempo retiene el momento de la crítica kantiana". ${ }^{32}$

${ }^{30}$ "Antwort", en Schilpp (ed.), op. cit., p. $7^{89}$.

si Cfr. Dufrenne y Ricoeur, op. cit., p. 126, n. 51.

s2 Ibid., p. 357. 


\section{Das Umgreifende}

La determinación de lo englobante, que ya adelantamos en parte, no hace sino reiterar los elementos kantianos que se descubren en la filosofía de Jaspers Un modo de aclarar esta noción, en efecto, es el siguiente: así como Kant demostró que espacio y tiempo no son objetos perceptibles, sino que, por el contrario, todo lo que se puede percibir aparece en ellos, del mismo modo, das Umgreifende no constituye un objeto de percepción o de pensamiento, sino más bien aquello en que (desde lo que, dice a veces Jaspers), se hace presente toda objetividad. ${ }^{33}$ Así, por ejemplo, el mundo, en cuanto Umgreifendes, "es el supuesto a priori y a su vez no objetivo, y el marco para el hecho de que puedan sernos dados objetos particulares".."

Ningún "ser-sabido" (objetivado), como gusta decir Jaspers, es el ser. Lo abarcante, lo englobante, es siempre el "anuncio" del ser que se anuncia en lo presente, pero sin revelar jamás su presencia. ${ }^{35}$

A esta vaga determinación de das Umgreifende añade Jaspers otro carácter de las Ideas: su función regulativa e impulsora del conocimiento objetivo, según se concluye del siguiente texto: "Una vez que he llegado a percatarme de lo englobante, se transforma el modo de mi saber acerca del ser del mundo. [...] En lo que respecta al contenido, el saber del mundo es por cierto siempre igual. Pero con todo saber está ligada una vivencia de significación, la mayoría de las veces no aclarada. Lo que yo sé de modo determinado y objetivo está soportado, en la vivencia de ese saber, por algo que yo experimento tan sólo por dicho saber, y que me impulsa a adquirir el saber, sin llegar a ser a su vez algo de que pueda tener saber [...] El modo en que ese sentido penetrante e impulsante soporta mi saber es causado por la presencia o la no presencia de das Umgreifende." 36

El pensar que apunta a la aclaración de lo englobante no puede fundar una ontologia - pues ello sería volver a hablar del ser-sabido, del fenómeno-, sino tan sólo instaurar "una nueva orientación fundamental en las posibilidades de relacionarse con el ser". Así como la Philosophie se abrla con una "orientación en el mundo" (con una mirada abarcadora y ordenadora de los modos de nuestro saber en el mundo y sobre el mundo), también el pensar de lo englobante se despliega en una amplia "orientación en el ser", esto es, en los diversos modos en que el ser (en sí incognoscible) se anuncia en lo englobante. ${ }^{37}$

s3 Cfr. Stegmüller, op. cit., p. 213.

34 Stegmüller, op. cit., p. 216.

35 Cfr. "Ueber meine Philosophie", en Rechenschaft und Ausblick, ed. cit., p. 353. Es obvio que hay cierta relación entre estas formulaciones y las de la teologia negativa 0 mística, en el sentido de que Dios (el Ser) no es nada de lo que es (de los entes objetivamente conocidos), como por ej. en el Pseudo Dionisio.

36 Von der Wahrheit, ed. cit., p. 105 .

37 Cfr. Knauss, op. cit., p. 155 . 
Estos diversos modos repiten en lo fundamental las Ideas kantianas -que también constituian los respectivos ejes de las tres partes de la Philosophie: el mundo, el alma (la libertad, la posible existencia), Dios (la Trascendencia)-, e impulsan al acto de trascender que llega al Umgreifendes aller Umgreifenden, al englobante que abarca todos los demás modos de lo englobante, es decir, a la Trascendencia. Pero la experiencia de lo absoluto se da junto con la de la esencial inadecuación de nuestro ser, en el fracaso a que nos condena la finitud: "Somos capaces de trascender, pero jamás llegamos a la Trascendencia. Seguimos siendo hombres. Ni siquiera abarcamos lo abarcador que somos nosotros mismos, pero lo experimentamos como abarcado." 38

\section{Trascender y Trascendencia en sentido estricto}

Allí donde el pensar no trasciende, no hay filosofía, afirma Jaspers. Alli donde se trasciende, empero, por cuanto la comunicación de tal acto debe enunciarse objetivamente, se corre siempre el peligro de aferrarse a las palabras, perdiendo en "lo dicho" el resorte vital propio de la libertad que trasciende. Pues la filosofía, en cuanto ese trascender, está en el límite y, en la medida en que no espera objeto alguno "más allá", sólo existe como acción interior transformadora de la existencia, nunca como "resultado". Por ello, ante la "conciencia en general", todo enunciado auténticamente filosófico es en realidad nulo, una nada, puesto que el filosofar es libertad y sólo para la libertad, como dice Jaspers con palabras que recuerdan a Marcel..$^{30}$ En última instancia, el paso decisivo del trascender se anula en cierto modo a sí mismo como pensamiento, ya que, en el límite, "es pensable que haya lo no pensable". ${ }^{40}$

Recurriendo a la nueva moción de das Umgreifende, Jaspers expresarfa lo mismo del siguiente modo: Nosotros somos el ser englobante, pero abarcados a la vez por el mundo y la Trascendencia. Lo otro, lo que no somos nosotros, es por una parte lo que llamamos mundo; pero nosotrò como libertad -y no como conciencia, existencia empírica o espiritu- llegamos también, por otra parte, a rozar una alteridad inefable a la que llamamos Trascendencia en sentido estricto. Aquello en lo cual y por lo cual somos empíricamente es el mundo; aquello por lo cual y en lo cual somos nosotros mismos y somos libres, es la Trascendencia. ${ }^{41}$

Para precisar más el sentido de este pensamiento, veamos un extenso pasaje de Jaspers: "Nosotros trascendemos lo englobante, es decir, sobre-

38 Die grossen Philosophen, p. go2.

se Cfr. Philosophie, 1, pp. 89 s.

to Philosophie, 1II, p. 88.

1. Cfr. Von der Wahrheit, p. 107. 
pasamos la objetividad determinada llegando a cerciorarnos de lo que la engloba; por ello, sería posible llamar trascendencia a cada uno de los modos de lo englobante [...] Sin embargo, Hamamos Trascendencia en sentido propio únicamente a lo englobante por antonomasia (das Umgreifende sthlechthin), a lo que engloba a todos los englobantes. [...] Frente a la trascendencia general que corresponde a cada uno de los englobantes, es ella la Trascendencia de todas las trascendencias. A esto corresponde el hecho de que los objetos del ser-mundo posean una doble transparencia:; ellos son fenómenos del ser englobante del mundo y están en relación con la Trascendencia. En cuanto fenómenos, y por la vía de su cognoscibilidad, permiten que se manifieste el ser abarcante del mundo. En cuanto cifras, hablan el lenguaje de la Trascendencia, equívoco, tan enfático como incognoscible. A través de la cognoscibilidad (de los fenómenos) resplandece das Umgreifende del ser-mundo. A través de su ser-cifra, se hace perceptible das Umgreifende de la Trascendencia."

"Trascendencia" es en definitiva, para Jaspers, tan sólo uno de los nombres con que intentamos circunscribir la experiencia de lo absoluto. Los nombres varian según la dimensión en que tenga lugar esa experiencia: la llamamos ser al intentar pensarla de modo abstracto; para el que vive efectivamente la relación con lo absoluto, ella es Trascendencia; pero si esa realidad apela a quien la experimenta, entonces la invocamos como Divinidad; y si entendemos esa Trascendencia como un Tú absoluto, como persona, la llamaremos Dios. Como ser, como Divinidad, como Dios, siempre esa Trascendencia es sólo "rozada" — dice Jaspers - y nombrada con nombres que parecen convertirla en objeto; pero la experiencia es tanto más decisiva cuanto menos desaparece la Trascendencia en el nombre y tanto más desaparecemos nosotros mismos en ella. Jaspers habla aqui el lenguaje del místico.

De todas maneras, el pensador trata de ordenar los modos en que nos acercamos a la Trascendencia. En el trascender formal el hombre se cerciora de la Trascendncia por medio de movimientos cognoscitivos metódicos, aun-

42 Von der Wahrheit, pp. 108 s. Según Fritz Kaufmann, esta noción de Trascendencia une la significación de Kant ("lo que yace más allá de la posibilidad del conocimiento humano") con los tonos de la teologfa negativa: "Totaliter aliter, mysterium tremendum del fundamento uno del ser. Esta conjunción, por lo demás, no sería extrafía en los escritos de Thomas Mann y Rilke; de este último cita Kaufmann el siguiente verso de su poema "Buddha": "Nichts ist so stumm / wie eines Gottes Mund" (Nada es tan mudo / como la boca de un Dios), Cfr. Kaufmann "Karl Jaspers und die Philosophie der Kommunikation", en Schilpp, op. cit., pp. 199 s., n. 19.

Al no advertir este maatiz mistico, el investigador puede bien decir que en Jaspers hay una teoría de la Trascendencia que a la postre admite que no hay Trascendencia, como por ej. F. J. von Rintelen, Philosophie der Endlichkeit als Spiegel der Gegenwart, Meisenheim/Glan, 1951, p. 369. Esta interpretación, lo mismo que la de Sciacca ("el salto en el vacio de Jaspers") en su libro La filosofia, hoy, Barcelona, 1956, pp. 205 ss., permanecerian sin embargo fuera del movimiento propio del filosofar de Jaspers, según dice Richard Wisser en Responsabilidad y cambio histórico, Buenos Aires 197o, pp. 44 ss. 
que destinados al fracaso - como sucede en la experiencia del límite en la orientación en el mundo-. Las referencias existenciales a la Trascendencia muestran otro modo de relación: la oposición o la entrega, la porfía y el abandono, la obediencia a la ley del día o la sumersión en la pasión de la noche, etc., hacen presente a la existencia, en su concreta historicidad, su relación con lo absoluto. Por último, la lectura del escrito cifrado, esto es, la conversión por cuya virtud todo objeto revela en su fondo la voz de la Trascendencia; aquí nosotros mismos llegamos a vivir como libertad. ${ }^{43}$.

También propone Jaspers una interesante aproximáción al concepto por esencia indefinible de la Trascendencia la cual retoma argumentaciones que ya habia esgrimido al referirse a Kant y a las "condiciones de posibiliedad de lo real". Dice al comienzo de la Metaphysik: Toda realidad se presenta en la correlación de algo objetivo y un sujeto dirigido activamente a ello. De este modo, la actividad de la conducta investigativa aprehende la realidad empirica como objeto del saber. Esta realidad se impone a la conciencia en general. La Trascendencia, en cambio, es otra realidad que se nos impone en cuanto somos existencia posible, libertad, que se manifiesta en la existencia empírica.

Ahora bien, "todo concepto determinado de realidad delimita con algo no real. Como posible existencia (por el contrario) pregunto por la realidad. absoluta. [...] La Trascendencia, en cuanto la realidad por la que pregunta la existencia, ya no puede ser interrogada con vistas a su validez general. Pues ella me alcanza como realidad sin posibilidad; como la absoluta realidad más allá de la cual nada hay; ante ella me quedo mudo. Lo que conozco como realidad empirica lo concibo como posibilidad, debido a las condiciones de su realización. [...] Lo que aprehendo como el ser de la mismeidad (la existencia) me es consciente como posibilidad de cuya realidad decide mi libertad. [...] La realidad de la Trascendencia; por el contrario, ya no es susceptible de una retraducción en términos de posibilidad; por ello, no es empírica; carece de la posibilidad aprehensible por nosotros desde la cual ella fuera real. Pero ello no se debe a un defecto, sino precisamente al hecho de que esa separación de realidad y posibilidad constituye el defecto de la realidad empírica, que siempre tiene otra cosa fuera de sí. Por lo mismo, además, tampoco es existencia: carece de la posibilidad de decisión, y no por defecto, sino a la inversa: la capacidad de decisión expresa la defectuosidad de la existencia en su existir concreto y empírico".

"Por tanto, alli donde encuentro la realidad sin su transformación en posibilidad, alli alcanzo la Trascendencia." 44

Ahora bien, el hecho de que la realidad de la Trascendencia no se ofrez-

4 Philosophie, III, pp. 8 s. 
ca directamente como cosa empírica ni como existencia, no debe inducirnos a creer que Jaspers la transfiere a un "más allá"; por el contrario, se trata de comprender cómo tal realidad trascendente únicamente puede manifestarse en el mundo. Por cierto, debido a que no será ya un objeto para la conciencia en general, cobrará necesariamente a los ojos de la actitud cognoscitiva "objetiva" el carácter de algo inconsistente, de una nada. En efecto, en cuanto manifestación de la Trascendencia, toda objetividad es evanescente (verschwindend) para la conciencia, puesto que "no es el ser entendido como consistencia o subsistencia, sino el ser de la Trascendencia como lenguaje para el ser de la libertad. Así como en general la existencia llega a sí misma al desaparecer lo que está ahí meramente de modo empírico, pero que no existe; así también, al dirigirse a la Trascendencia, sólo lo hace en objetos que como tales no tienen consistencia alguna para la conciencia". En tal sentido, pues, lo que la metafísica considera como objeto, no es ese objeto mismo, sino un simbolo. Con ello, por tanto, la búsqueda metafísica de la Trascendencia culmina en la teoria de las cifras. ${ }^{45}$

\section{La teoria de las cifras}

El "contacto", como suele decir Jaspers, de la existencia con la absoluta realidad (sin posibilidad) de la Trascendencia, despierta la conciencia de la finitud, de no ser yo mismo el absoluto origen de mis decisiones. También aquí se llega a la conciencia del límite: del "otro lado" de lo que limita la libertad se encuentra la facticidad; la existencia se sabe "donada" por la Trascendencia. En el fracaso -otra palabra para la conciencia de la finitud- la existencia pércibe el lenguaje enigmático de la Trascendencia —dice Johannes Thyssen-; ${ }^{46}$ su desciframiento es tarea de cada uno, no hay código universal, no hay ontologia.

La filosofía de Jaspers, por tanto, concluiría en la aserción de que el ser que se manifiesta en el mundo no es lo absoluto, sino que está lacerado por contradicciones insuperables; y ello en todos los órdenes de la vida: los valores positivos no se realizan sino al precio de valores negativos no queridos; la libertad está siempre oscurecida por la necesidad; la comunicación se

45 Philosophie, III, p. 15. Como ya señalamos al comienzo, Jaspers "introduce" abruptamente la Trascendencia en su filosoffa, al definir la existencia. En virtud de ello, y basándose en Heidegger, Hans Kunz opina que quizá pudiera llevarse a cabo la “aclaración de la existencia" prescindiendo de la Trascendencia. Kunz se basa primordialmente en el análisis del problema de la muerte. Como siempre, Jaspers responde a su critico reprochándole el que tome sus pensamientos -que "sólo recuerdan o preparan una experiencia como realización de la libertad"- como si fueran "doctrinas" o "saberes" filosóficos, más aún, cientificos, con lo cual yerra su argumentación. Cfr. Hanz Kunz, "Versuch einer Auseinandersetzung mit der Transzendenz bei Jaspers", y la correspondiente "Antwort" de éste, ambas en el volumen de Schilpp, pp. 493-514 y 813-820 respectivamente.

46 Cfr. J. Thyssen, "Der Begriff des Scheiterns bei K. Jaspers", en el volumen de Schilpp, p. 294 s. 
ve de continuo desplazada por la soledad; la existencia posible destruye sus posibilidades al encarnarse en la existencia empírica, etc. El fracaso seria en definitiva la última situación límite tanto en el plano de la acción como en el del conocimiento. Pero Jaspers no se detiene en la mera descripción de esta ineludible circunstancia; la misma situación límite del fracaso se convierte en decisiva cifra para interpretar todas las otras cifras. $Y$ esto sucede en la medida en que tal experiencia me lleva a renunciar a la ilusión de un sistema total del conocimiento y de la acción, cuando "yo asumo y trasciendo el mundo como cuerpo de mi libertad y como cifra de la Trascendencia"."

Con reminiscencias de las vías analogicas y negativas de algunas doctrinas medievales, Jaspers descifra toda objetividad y toda experiencia en la doble perspectiva del fracaso y la presencia, en una suerte de "ontologfa" -opinan Dufrenne y Ricoeur_ tendida entre los dos abismos de una Trascendencia que ya no revelaria ninguna inmanencia y un pantefsmo en el que se absorberia la trascendencia de lo totalmente otro. 48

Para comprender el "mecanismo", por asi decir, de la teoria de las cifras, es menester recordar que todo filosofar, según Jaspers, es un pensar de o desde das Umgreifende. En efecto, como señala Knauss, ya los primeros pensadores griegos partían de esa previa posición de la totalidad: todo es agua, todo es fuego, etc. La posterior interpretación del significado de esa totalidad es en cierto modo relativa al momento histórico cultural. Lo importante en este contexto es el hecho de que para que se pueda pensar el ser como agua, fuego, etc. previamente hubo de ser pensada la totalidad, de alguna manera, bajo el concepto de ser. "El comienzo del filosofar no reside propiamente en los primeros fragmentos que nos han sido trasmitidos, sino en el pensar anterior a ellos. Frente a eso, la cualificación más detallada del ser casi significa ya, nuevamente, una decadencia del pensar." 49 Jaspers quizá admitiria su cercanía a esos "modelos", que pueden ejemplificarse en el concepto de ápeiron de Anaximandro, que él ha traducido en cierto modo en das Umgreifende y en la Periechontologie. Así dice: "Nuestro camino es la aclaración de das Umgreifende que buscamos como aquello que cobija en sí todo origen, pero que sin embargo jamás puede llegar a ser objeto de modo adecuado"; camino que, para diferenciarlo del tradicional de la ontología, lo llamamos —concluye- "Periechontologie".so

47 Dufrenne y Ricoeur, op. cit., pp. 194 y 286. Cfr. también Thyssen, op. cit., p. 299.

48 Dufrenne y Ricoeur, p. 375. Se habla aqui de "ontologia", por cierto, pero en un sentido muy especial que no contradice nuestra afirmación de que Jaspers niega la ontologia. Es "ontologia" en cuanto se habla del ser; pero en la medida en que todo enunciado acerca del mismo es tomado como mero símbolo o cifra de su presencia, es obvio que no estamos ante una disciplina ontológica en el sentido tradicional, pues la cifra sólo es "inteligible" para una existencia individual y concreta que se compromete al descifrarla; jamás es enunciable su código de modo universal y necesario,

40 G. Knauss, op. cit., p. 131.

50. Von der Wahrheit pp. 159 s. Knauss alude a la formulación atribuida a otro pasaje 
Vista desde esta perspectiva, todas las doctrinas filosóficas cobran una nueva significación. En efecto, tanto las milenarias metafísicas de los elementos como las grandes doctrinas sobre el ser "consisten en una escritura cifrada que los filósofos proyectan desde la presencia de lo englobante, para aclarar el ser y aclararse a sí mismos", pero estas "cifras" fueron luego "erróneamente tomadas como un determinado ser-objeto entendido como el verdadero ser". pix

Con la teoría de las cifras reaparece en Jaspers, en otro nivel, su interpretación del pensamiento de Kant. Esto es claro si volvemos a plantear la cuestión desde un comienzo. En la orientación en el mundo, el hombre, en cuanto conciencia en general convierte toda realidad en objeto de investigación; en tal sentido es la realidad manifestación o fenómeno de algo que teóricamente subyace a dicha manifestación. Ahora bien, ni la existencia en sentido estricto, ni la Trascendencia, pueden ser objetos de investigación. Pero considerados en el modo de pensar que trasciende a la conciencia en general, los mismos fenómenos delatan una nueva dimensión: son ahora símbolos, objetos que se desvanecen ante la mirada metafísica. En el primer caso, en el plano de la ciencia, el objeto es fijado en sí mismo; en el segundo, no es tomado como tal objeto, sino como aquello en que "toma la palabra". lo que jamás puede aprehenderse de modo directo y así se manifiesta simbólicamente en toda experiencia de objetos.

El símbolo, en este sentido, no remite a otra cosa que también podría aparecer sin ese símbolo: en él se hace presente "otro" que sin ese medio no pódría aludirnos en modo alguno. La mirada metafísica, dice Jaspers, revela como intrínsecamente contradictorio al objeto de la conciencia en general - de all las antinomias kantianas, en virtud de las cuales se alcanza indirectamente en lo objetivo aquello que jamás puede llegar a ser objeto. ${ }^{52}$

Además de las dos "funciones" señaladas —objeto de conocimiento y objeto metafísico o simbolo puede indicarse aún la de signum existencial. En efecto, en el objeto se hace presente una posibilidad de ser uno mismo, la que presupone las dos anteriores funciones (el conocimiento y el símbolo); todo saber y todo símbolo se transforman en invocación, en el llamado a la más propia posibilidad de ser yo mismo.

Estas reflexiones de Jaspers parecen recuperar una línea muy cara a los alemanes, basada en la distinción kantiana entre conceptos e Ideas. Quizá ryecordara Jaspers la versión aforística de estos pensamientos, tal como se

con la misma expresión (Die Fragmente der Vorsokratiker, $8^{\mathfrak{a}}$ ed. compil. por W. Kranz, Berlín, 1956, T. I, p. 84). Pero Jaspers, cuando se refiere a Anaximandro, curiosamente no menciona este término del que, según la plausible exposición de Knauss, provendria su idea de la "periejontologia".

51 Jaspers, Einführung in die Philosophie, München, 1969, p. 34.

52 Cfr. Von der Wahreit, p. $256 s$. Véase también Jean Paumen, Raison et existence chez Karl Jaspers, Bruselas, 1958, p. 218. 
encuentra en Goethe. Este, al distinguir entre metáfora y simbolo, escribe: "El verdadero simbolismo es aquel donde lo particular representa lo universal, pero no como sueño y sombra, sino como revelación viva e instantánea de lo ininvestigable". "El símbolo - aclara en otra oportunidad- transforma el fenómeno en Idea; la Idea, en imagen; pero de tal manera que la Idea permanece en la imagen siempre infinitamente activa e inalcanzable, y aun cuando se le expresara en todas las lenguas seguiria siendo sin embargo inexpresable." ss

El símbolo de que habla Jaspers encuentra una denominación más adecuada con la noción de cifra, pues ésta alude a la necesidad de su desciframiento por cada existencia histórica. Pero aunque la apelación es inmediata y personal, Jaspers admite una cierta estructuración de ese lenguaje inmediato de la Trascendencia en un segundo lenguaje, donde los contenidos aparentemente incomunicables se sedimentan en los mitos, las revelaciones de un más allá, etc. Por último, el pensamiento puede remontar hasta el origen este lenguaje mediato e intuitivo, aprehendiendo en la especulación metafísica aquello que en verdad es incognoscible e inefable, pero que llega a ser un tercer lenguaje. Por tanto, bien puede decirse que el filósofo "lee el escrito cifrado originario a medida que va escribiendo uno nuevo: piensa la Trascendencia por analogía con la existencia empírica que le es intuitiva".st

Pero la originalidad de la teoria de las cifras de Jaspers reside en este hecho señalado por Kurt Hoffman: mientras casi todos los filósofos que llegan a una visión totalizadora de la historia de la filosofía tienden a absolutizar su propio sistema, rechazando por ende los que se le oponen, Jaspers, por el contrario, admite la cifra, el núcleo verdadero de todo gran pensamiento, pero rechazando su pretensión de validez universal, objetiva y definitiva. De tal modo, en esta "filosofía de la filosofia", Jaspers "considera consecuentemente su propio pensamiento como una metáfora filosófica entre otras, y este hecho es tan necesario como problemático"..55

La doctrina de las cifras es la más clara expresión de un pensar que, en todo momento, trata de preservar el origen autónomo e independiente del quehacer filosófico, especialmente frente a los dos ámbitos que históricamente pretenden reducirlo a sus propias dimensiones: la ciencia y la religión. El "objeto" de la filosofía, por una parte, no es susceptible de un conocimiento válido para la conciencia en general. En tal sentido, si bien en la actualidad la auténtica filosofía no puede prescindir de los resultados de la ciencia - como expresamente reconoce Jaspers-, ella apunta a otra verdad, ya no cientifica. Por otra parte, la filosofia se aproxima al ámbito

53 "Maximen und Reflexionen", en Goethes Werke, edit. por Heinrich Kurz, Leipzig, 8. a., t. XIII, pp. 695 y 741 respectivamente.

of Cfr. Philosophie, III, pp. 134 s.

ss Kurt Hoffman, "Die Grundbegriffe der Philosophie K. Jaspers", en el volumen de Schilpp, p. 96. 
de la fe. Pero la doctrina de las cifras impide que se confunda la "fe filosófica" con la creencia en una revelación histórica objetiva hecha por Dios, de una vez y para siempre, para todos los hombres. En conclusión, pues, la religión será "falsa como dogma, verdadera como mito" (como cifra); del mismo modo, la filosofía "científica", la ontología en el lenguaje de Jaspers, "será falsa en cuanto ciencia, pero verdadera como cifra especulativa". ${ }^{56}$

Pero en este punto urge preguntarse, nuevamente, hasta qué punto Jaspers, al alejarse de la base kantiana en que inicialmente se apoyara su pensamiento, no corre el riesgo de edificar "en el aire", falto de aquellos sólidos cimientos de la filosofía crítica. En efecto, aferrándose a la letra de la ya citada confesión de Kant, Jaspers suprime el saber para hacer lugar a la fe. Pero a una fe que no puede comunicarse sino simbólicamente, y que parece condenar a una insalvable soledad. El filósofo de la comunicación, pues, termina en la inevitable narración de su propia búsqueda de lo absoluto. La filosofía es devorada por la autobiografía.

Untversidad de la Plata

Mario A. Presas

56 Paul Ricoeur, Gabriel Marcel et Karl Jaspers. Philosophie du Mystère et Philosophie du Paradoxe, Paris, 1947, p. 281. El mismo autor, en su trabajo "Philosophie und Re-" ligion bei K. Jaspers" (vol. Schilpp, pp. 6.17 s.) expresa: la cifra remplaza en Jaspers al milagro; la contemplación metafísica, a la plegaria; la comunicación, a la Iglesia. En el libro que escribió en colaboración con. Dufrenne se afirma que la doctrina de las cifras es el punto extremo de una secularización de los dogmas cristianos.

Con una intención más conciliatoria, Bernard Welte compara la "fe filosófica" de Jaspers con el pensamiento de Santo Tomás de Aquino. Cfr. La foi philosophique chez Jaspers et Saint Thomas d'Aquin, Paris, 1958. 